\title{
VORTICITY DYNAMICS AND TURBULENCE MODELS FOR LARGE-EDDY SIMULATIONS
}

\author{
Georges-Henri Cottet $^{1}$, Delia Jiroveanu ${ }^{1}$ and Bertrand Michaux ${ }^{1}$
}

\begin{abstract}
We consider in this paper the problem of finding appropriate models for Large Eddy Simulations of turbulent incompressible flows from a mathematical point of view. The Smagorinsky model is analyzed and the vorticity formulation of the Navier-Stokes equations is used to explore more efficient subgrid-scale models as minimal regularizations of these equations. Two classes of variants of the Smagorinsky model emerge from this approach: a model based on anisotropic turbulent viscosity and a selective model based on vorticity angles. The efficiency of these models is demonstrated by comparisons with reference results on decaying turbulence experiments.
\end{abstract}

Mathematics Subject Classification. 35Q30, 81T80.

Received: September 16, 2002. Revised: November 19, 2002.

\section{INTRODUCTION}

The most encountered flows in nature and engineering applications are turbulent, and their prediction represents at present an extremely challenging field of research.

The Navier-Stokes equations are generally accepted as providing an accurate model for the incompressible motion of fluid flows. From a mathematical point of view, except for very simple flows, there is no analytical solution for these nonlinear equations. Their well posedness in the classical sense is limited to a certain range of parameters or short time intervals. In the three-dimensional case the existence for all time of strong solutions or the uniqueness of weak solutions are classical examples of persisting open problems.

Concerning numerical techniques, a first natural class of methods are the so-called Direct Numerical Simulations (in short DNS), which assume the computation of all temporal and spatial scales. Since one of the main features of a turbulent flow is the presence of a large span of dynamically important scales, from the largest, imposed by the boundaries or geometry, to the smallest dissipative scales, it is clear that DNS are limited to low Reynolds number flows by the available computational resources. Consequently, DNS are and will be, at least in the foreseeable future, mainly used to study turbulence physics for simple reference cases and/or at low Reynolds numbers and to validate turbulence models. An alternative to DNS is the statistical approach based on the idea that turbulence consists of random fluctuations of various flow properties. Reynolds (1895) introduced the averaging procedure, in which all quantities are expressed as the sum of a mean (resolved) part and a fluctuation (residual) component. Time, ensemble or spatial averaging is usually used in function of the

\footnotetext{
Keywords and phrases. Navier-Stokes equations, large eddy simulation, subgrid-scale modeling, Smagorinsky model, selective anisotropic model.

1 LMC-IMAG, Université Joseph Fourier, BP 53, 38041 Grenoble Cedex, France.

e-mail: Georges-Henri.Cottet@imag.fr, Delia.Jiroveanu@imag.fr, Bertrand.Michaux@imag.fr
} 
considered application. Time averaging is employed in the Reynolds Averaged Navier-Stokes (in short RANS) models which are very appropriate for statistically stationary turbulence. This last method yields in many cases excellent results [45]. However, it does not work well for separated flows in general [49]. For an accurate computation of transition to turbulence, one has to know in advance where it occurs which is not simple to determine.

Since, most often, the engineers are only interested in the evolution of large spatial eddies which contribute the most to turbulent stresses and fluxes, it makes sense to apply a spatial averaging to the Navier-Stokes equations. The idea of the Large Eddy Simulation (in short LES) technique is to compute directly only the scales at larger than some cutoff wavenumber, in general given by the grid resolution, and to model the smallest scales via a subgrid stress tensor. It assumes that the small scale turbulence structures are not significantly dependent on the particular geometry of the flow and thus are appropriate for general modeling. With this method, one is able to handle both transition and turbulent regimes without specifying explicitly where the transition occurs.

By averaging the flow, we obtain a set of partial differential equations (the LES equations) for the resolved velocity $\overline{\mathbf{u}}=\left(\bar{u}_{1}, \bar{u}_{2}, \bar{u}_{3}\right)$ and pressure $\bar{p}$, conventionally written as ${ }^{1}$

$$
\partial_{t} \bar{u}_{i}+\partial_{j}\left(\bar{u}_{i} \bar{u}_{j}\right)=\bar{f}_{i}-\partial_{i} \bar{p}+\nu \partial_{j j}^{2} \overline{u_{i}}-\partial_{j} \tau_{i j} \quad i, j=1,2,3
$$

and

$$
\partial_{i} \bar{u}_{i}=0 \quad i=1,2,3 .
$$

$\nu$ is the kinematic viscosity and the tensor $\tau$ defined by

$$
\tau_{i j}=\overline{u_{i} u_{j}}-\bar{u}_{i} \bar{u}_{j}
$$

is the result of filtering the nonlinear term in the Navier-Stokes equations and describes the effects of the subgrid scales quantities on the resolved velocity. This tensor, usually referred to as the subgrid-scale stress tensor (SGS), has to be modeled in terms of the resolved quantities, in order to close the LES equations (1).

The main issue in SGS modeling is the formulation of a physically realistic description of the dynamic interaction between the resolved scales and the subgrid scales. This supposes a good understanding and knowledge of turbulence mechanisms. One of the most important results in turbulence physics is the Kolmogorov theory [26] which assumes that turbulence generation occurs mainly at the smallest scales, leaving a range of scale sizes (the inertial subrange) over which external influences (body forces or frictional boundaries) and viscosity are negligible. These scales get turbulent energy by nonlinear transfer of the energy from the larger scales and lose energy by subsequent transfer to smaller scales (the energy cascade). This fragmentation process stops at the smallest scale (called the Kolmogorov dissipation scale) where the energy is dissipated by kinematic viscosity in the form of heat. Based on this theory, physicists recommend modeling the SGS tensor by dissipative terms which should have a compulsory effect in the energy cascade, playing the role of missing smaller eddies. LES equations equipped with proper SGS models are expected to allow the computation of coherent structures at coarser spatial resolutions than those required by DNS.

Several subgrid-scale models are commonly used in large eddy simulations of turbulent flows. The most currently used are eddy-viscosity models which are based on the following linear dependence between the SGS tensor and the resolved strain rate tensor $\bar{S}_{i j}=\frac{1}{2}\left(\partial_{j} \bar{u}_{i}+\partial_{i} \bar{u}_{j}\right)$

$$
\tau_{i j}-\frac{1}{3} \tau_{k k} \delta_{i j}=-2 \nu_{t} \bar{S}_{i j}
$$

where $\delta_{i j}$ is the Kronecker symbol and $\nu_{t}$ is a coefficient called eddy viscosity. From a physical point of view, this hypothesis assumes that the small scales have a purely dissipative effect on the resolved scales, which is

\footnotetext{
${ }^{1}$ Here and in the sequel we use the notation $\partial_{j} u_{i}=\frac{\partial u_{i}}{\partial x_{j}}$ and we adopt the implicit summation for the repeated indices.
} 
just a first approach in the LES modeling. The trace term in (3) is usually absorbed in the pressure term, introducing in (1) a modified pressure given by $\bar{P}=\bar{p}-\frac{1}{3} \tau_{k k}$.

Among the eddy viscosity models, the Smagorinsky model originally proposed for atmospheric turbulence [44] is the most common tool. This model assumes a linear relationship between $\nu_{t}$ and the strain rate $|\mathbf{S}|=$ $\sqrt{2 S_{i j} S_{i j}}$. It has been applied extensively to engineering applications, often giving fair agreement with experiments and observations. However, it turns out to be too dissipative, especially in laminar regimes and close to walls, and thus does not work satisfactorily for the computation of the transition to the turbulence. Dynamical models [23] were proposed to correct the excessive dissipation of the Smagorinsky model by allowing the constant in front $|\mathbf{S}|$ to vary in space and time. This constant is dynamically computed in terms of the resolved scales during the simulation. These models give the correct asymptotic behavior for the dissipation near the walls. One drawback of the dynamical models however is that, in order to provide strict dissipation which means a positive constant, it in general requires assuming flow homogeneity in at least one direction and thus does not readily apply to general geometries. An alternative approach to remove the excessive dissipation in the Smagorinsky model is to either apply it with strain rates based on fields where large scales have first been filtered out [20] or, selectively, only in flow regions where vorticity undergo large variations [18]. We will describe later in more details this selective Smagorinsky model.

For the original Smagorinsky model, the filtering operation does not appear explicitly, being dependent of the given grid resolution or the numerical method employed for the simulation. In contrast to implicit filtering, anisotropic models assume an explicit formulation of the filtering operation. The most natural anisotropic model is the gradient model [48] based on a Taylor series approximation of the Fourier transform of the filter. This model gave in a priori tests, that is in experiments where actual measured values of $\tau_{i j}$ are compared to values predicted by the models, good correlations with high resolution DNS or experimental data, as reported in $[4,35]$. A posteriori tests, that is experiments where results obtained by low resolution LES are compared to filtered results from high resolution DNS, showed that this model is not dissipative enough. The reason is that, since in this model $\nu_{t}$ is a tensor rather than a scalar, it may produce backscatter as well as dissipation which could lead to numerical instabilities [48]. One way to overcome this deficit is to apply a clipping procedure which consists of ignoring the subgrid-scale term at all points where its contribution to energy balance would be positive. The drawback of this technique is that it does not take into account that dissipation and backscatter coexist at every point along different strain directions of the flow. Hence, it appears that the resulting models do not dissipate enough energy. This is why the gradient model is usually combined with a purely dissipative model (for example, a Smagorinsky model), in the so-called mixed model, but this new formulation has the deficit to retain the excessive dissipation of the added model [7]. To avoid this dilemma, Cottet [14,17] has proposed an implementation of the gradient model which identifies in the gradient viscosity tensor the direction along which the model does provide strict dissipation. This allows to derive an anisotropic model which at the same time retains the good correlations of the gradient model and overcomes its stability problems. We will come back later to this model.

An alternative to the gradient model, proposed by Galdi and Layton [22], consists of replacing the Taylor series approximation with a rational Padé approximation of the Fourier transformation of a Gaussian filter. This technique leads to a SGS model referred to, in literature, as a RLES (rational LES) model. Numerical studies on this model are reported in [24].

A recent approach introduced by Holm and Marsden [21] proposes the derivation of a new class of SGS models, called alpha-models, which use geometrical methods. The averaged Lagrangian method can be viewed as a filtering operation. The effect of eddies smaller than a given length scale is parameterized by nonlinear diffusion terms in order to moderate the forward energy cascade. This moderation enhances the stability and regularity of the alpha fluid models, as the mathematical results of $[21,37]$ show. Numerical studies with these models have been performed with success [40], but it seems that they have some limitations (for more details see [25]). However, this mathematical approach offers a new basis for LES modeling and computational fluid dynamics. 
In this paper, we also consider the LES closure problem from a mathematical point of view. The closure SGS models are usually derived from physical arguments based on the energy cascade. From a mathematical standpoint, these models are closely linked to deconvolution models [46] (see also [19] for SGS models viewed from the angle of Nonlinear Galerkin methods) and could be interpreted as minimal regularizations for the Navier-Stokes equations, allowing smoother solutions. Since the LES models are supposed to compute the solutions to averaged equations, the Navier-Stokes equations supplemented by the corresponding SGS models should ensure the existence and uniqueness of smooth solutions for all time. We prove in this paper that adding a Smagorinsky term is indeed sufficient to obtain the regularity and the uniqueness of the solution. Since in practice, as we already said, this model turns out to be too dissipative, we focussed our interest on the problem of finding minimal models (in the sense of the less dissipative) capable of assuring the regularity of the solution. In doing so, the vorticity form of the three-dimensional Navier-Stokes equations is a useful tool to understand the mechanisms for the possible lack of regularity of its solutions, and thus to propose appropriate smoothing factors. The vorticity stretching term in these equations, which vanishes in $2 \mathrm{D}$, is responsible for the possible vorticity blow up, which is a prerequisite for singularity in the Navier-Stokes equations [2]. SGS models can thus be viewed as dissipation terms to counterbalance this stretching term.

The aim of this paper is to revisit, on the basis of this remark, the selective and anisotropic SGS models mentioned above, and to evaluate numerically their efficiency in homogeneous isotropic turbulence experiments.

This paper is organized as follows. In Section 1, we first establish in a simple manner the well posedness of the three-dimensional Navier-Stokes equations with a Smagorinsky term. Then in Section 2, we recall the vorticity form of the Navier-Stokes equations. We use this formulation to interpret the anisotropic model. We then turn to the selective Smagorinsky model and show that it can be understood in the light of a necessary condition for singularity which was established by Constantin and Fefferman [11] and recently improved by Beirão da Veiga and Berselli [3]. In Section 3 we demonstrate the modeling capabilities of both selective Smagorinsky and anisotropic models on the decaying homogeneous turbulence test case. We finally draw in Section 4 some conclusions and directions for future work.

\section{Mathematical analysis of the Smagorinsky MODEL}

\subsection{The equations}

In spite of its simplicity, the Smagorinsky model [44] is still extensively used in practice and it serves as basic tool for the developments in the LES modeling. Based on the Boussinesq hypothesis (3), the Smagorinsky subgrid scale stress tensor is assumed to be proportional to the strain rate tensor of the resolved field through the eddy viscosity defined by ${ }^{2}$

$$
\nu_{t}^{\mathrm{sm}}=\left(C_{\mathrm{s}} \Delta\right)^{2}|\overline{\mathbf{S}}|
$$

where $\Delta$ is a subgrid characteristic length scale, $|\overline{\mathbf{S}}|=\sqrt{2 \bar{S}_{i j} \bar{S}_{i j}}$ and $C_{\mathrm{s}}$ is a nondimensional constant which has to be calibrated. Its value may vary with the type of the flow and the Reynolds number. Values ranging from 0.1 to 0.24 have been suggested in the literature [43].

Thus, for an incompressible fluid which fill a domain $\Omega \subset \mathbb{R}^{3}$, with sufficiently smooth boundary $\partial \Omega$, the LES-Smagorinsky equations read as

$$
\partial_{t} \bar{u}_{i}+\partial_{j}\left(\bar{u}_{i} \bar{u}_{j}\right)=f_{i}-\partial_{j} \bar{P}+2 \partial_{j}\left[\left(\nu+C_{\mathrm{s}}^{2} \Delta^{2}|\overline{\mathbf{S}}|\right) \bar{S}_{i j}\right] \quad i, j=1,2,3
$$

and

$$
\partial_{j} \bar{u}_{j}=0
$$

\footnotetext{
${ }^{2}$ Throughout this paper, we will use bold face notations for vectors and vector valued functions.
} 
where $\overline{\mathbf{u}}=\left(\bar{u}_{1}, \bar{u}_{2}, \bar{u}_{3}\right)$ is the resolved velocity field, $\bar{P}$ the modified pressure, $\nu$ the kinematic viscosity and $f$ the external volume force.

Remark that adding a dissipative Smagorinsky term implies a modification of the molecular viscosity such that one compensates for the missing part of non-linear interaction terms binded to the small scales.

For the sake of simplicity, in the remaining of the paper we will drop the overbar notation for the resolved quantities. Denoting

$$
\mathcal{T}(\mathbf{S})=2\left(\nu+\nu_{1}|\mathbf{S}|\right) \mathbf{S}
$$

with $\nu_{1}=\left(C_{\mathrm{s}} \Delta\right)^{2}$, we are now concerned with the resolution of the following initial value problem:

Find $\mathbf{u}(\mathbf{x}, t)$ and $P(\mathbf{x}, t)$ solutions of

$$
\left(\partial_{t}+\mathbf{u}(\mathbf{x}, t) \cdot \nabla\right) \mathbf{u}(\mathbf{x}, t)=\mathbf{f}(\mathbf{x}, t)-\nabla P(\mathbf{x}, t)+\operatorname{div} \mathcal{T}(\mathbf{x}, t) \quad \text { for } \quad(\mathbf{x}, t) \in \Omega \times(0, T)
$$

and

$$
\operatorname{divu}(\mathbf{x}, t)=0 \quad \text { for } \quad(\mathbf{x}, t) \in \Omega \times(0, T)
$$

with the initial condition

$$
\mathbf{u}(\mathbf{x}, 0)=\mathbf{u}_{\mathbf{0}}(\mathbf{x}) \quad \text { for } \quad \mathbf{x} \in \Omega
$$

and the Dirichlet boundary condition

$$
\mathbf{u}(\mathbf{x}, \cdot)=0 \quad \text { for } \quad \mathbf{x} \in \partial \Omega
$$

Note that the mathematical results presented in the following can be easily extended to the periodic case, which corresponds to the numerical results presented in the last section, with a proper setting of functional spaces and operators.

\subsection{Functional setting of the equations}

Let us first introduce some standard notations and function spaces which will be used in the following analysis.

We denote

$$
\begin{aligned}
& \mathcal{V}=\left\{\varphi \in \mathcal{D}(\Omega)^{3}, \operatorname{div} \varphi=0\right\} \\
& H=\text { the closure of } \mathcal{V} \text { in } L^{2}(\Omega)^{3} \\
& V=\text { the closure of } \mathcal{V} \text { in } W^{1,3}(\Omega)^{3}
\end{aligned}
$$

where $L^{2}(\Omega)$ is the space of functions which are square integrable over $\Omega$ with respect to the Lebesgue measure and $W^{1,3}(\Omega)$ is the $L^{3}$ Sobolev space. It is known that $H$ is a Hilbert space with respect to the inner product

$$
(\mathbf{u}, \mathbf{v}):=\int_{\Omega} \mathbf{u} \cdot \mathbf{v} \mathrm{d} \mathbf{x} \quad \text { for } \quad \mathbf{u}, \mathbf{v} \in H
$$

and the corresponding norm $\|\mathbf{u}\|_{2}=(\mathbf{u}, \mathbf{u})^{1 / 2}$ and $V$ is a reflexive Banach space endowed with the norm

$$
\|\mathbf{u}\|_{1,3}:=\left[\int_{\Omega}|\nabla \mathbf{u}|^{3} \mathrm{~d} \mathbf{x}\right]^{1 / 3} \quad \text { for } \quad \mathbf{u} \in V .
$$


We will use the notation $V^{\prime}$ for the dual space of $V,\|\cdot\|_{V^{\prime}}$ for the induced norm and $\langle\cdot, \cdot\rangle$ for the duality product. For spaces of functions which depend on both time and space variables, we will frequently use the two following spaces

- $C([0, T] ; X)$ the space of continuous functions $u:[0, T] \rightarrow X$, where $X$ is a Banach space with the norm denoted by $|\cdot|_{X}$ and,

$-L^{p}(0, T ; X)$ the space of strongly measurable functions $\left.u:\right] 0, T[\rightarrow X$ with a finite norm

$$
|u|_{L^{p}(0, T ; X)}^{p}:=\int_{0}^{T}|u(t)|_{X}^{p} \mathrm{~d} t<\infty .
$$

In the case $p=\infty$ the norm is defined by

$$
|u|_{L^{\infty}(0, T ; X)}:=\operatorname{ess} \sup _{t \in] 0, T[}|u(t)|_{X} .
$$

Finally, we will denote by $|\cdot|_{p}$ the usual norm in $L^{p}(\Omega)$.

We recall that, due to the continuous embeddings $V \hookrightarrow H \hookrightarrow V^{\prime}$, one can prove [36] that:

$$
\left\{\mathbf{u} \in L^{p}(0, T ; V) ; \frac{\mathrm{d} \mathbf{u}}{\mathrm{d} t} \in L^{p^{\prime}}\left(0, T ; V^{\prime}\right)\right\} \hookrightarrow C([0, T] ; H), \text { where } \frac{1}{p}+\frac{1}{p^{\prime}}=1 .
$$

We recall also a useful lemma, due to Aubin and Lions:

Lemma 1.1. Let $X$ be a Banach space and $X_{0}, X_{1}$ two reflexive, separable Banach spaces. If we assume that

$$
X_{0} \hookrightarrow \hookrightarrow X \hookrightarrow X_{1}
$$

the first embedding being compact, then we have the following embedding

$$
\left\{v \in L^{\alpha}\left(0, T ; X_{0}\right), \frac{\mathrm{d} v}{\mathrm{~d} t} \in L^{\beta}\left(0, T ; X_{1}\right)\right\} \hookrightarrow \hookrightarrow L^{\alpha}(0, T ; X),
$$

where $1<\alpha, \beta<\infty$.

The proof of this lemma can be found in [34].

In this context, we consider the weak formulation for the problem (7)-(10). It is obtained by multiplying the momentum equation (7) by a test function and integrating by parts, yielding the problem which will be referred in the sequel as:

Problem $(\mathcal{S})$. For $\mathbf{f} \in L^{3 / 2}\left(0, T ; V^{\prime}\right)$ and $\mathbf{u}_{0} \in H$ given, find $\mathbf{u}$ satisfying

$$
\begin{gathered}
\mathbf{u} \in C([0, T] ; H) \cap L^{3}(0, T ; V) \quad \text { with } \quad \frac{\mathrm{d} \mathbf{u}}{\mathrm{d} t} \in L^{3 / 2}\left(0, T ; V^{\prime}\right), \\
\left\langle\frac{\mathrm{d} \mathbf{u}}{\mathrm{d} t}, \mathbf{v}\right\rangle+\sum_{i, j=1}^{3} \int_{\Omega} \mathcal{T}_{i j}(\mathbf{S}(\mathbf{u})) S_{i j}(\mathbf{v}) \mathrm{d} \mathbf{x}+\int_{\Omega}(\mathbf{u} \cdot \nabla) \mathbf{u} \cdot \mathbf{v} \mathrm{d} \mathbf{x}=\langle\mathbf{f}, \mathbf{v}\rangle \quad \forall \mathbf{v} \in V
\end{gathered}
$$

and the initial condition

$$
\mathbf{u}(0)=\mathbf{u}_{0} .
$$




\subsection{Global existence and uniqueness}

In this section we address the problem of global existence and uniqueness of weak solutions to problem $(\mathcal{S})$. This problem was already considered by Ladyszenskaya [29]. For the sake of completeness, we give here a simpler proof.

Theorem 1.2. Let $\mathbf{u}_{0} \in H$ and $\mathbf{f} \in L^{3 / 2}\left(0, T ; V^{\prime}\right)$. Then for any $T>0$ the problem $(\mathcal{S})$ has a unique weak solution on $[0, T]$. Moreover, if $\mathbf{u}_{0} \in V$ then the unique weak solution is in $L^{\infty}\left(0, T ; W^{1,3}(\Omega)^{3}\right)$.

Proof. To prove the existence of a weak solution we used a classical Galerkin method. We omit it, since it is straightforward from the proof done in Lions [34] based on the compactness method. A complete demonstration can be found in $[25]^{3}$ (see also [41]). We only present here the proof of uniqueness.

Let us suppose that there exist two weak solutions $\mathbf{u}$ and $\mathbf{v}$ to problem $(\mathcal{S})$, with the same initial condition $\mathbf{u}_{0} \in H$ and let $\mathbf{w}=\mathbf{u}-\mathbf{v}$. After subtracting the weak formulation for $\mathbf{v}$ from the one for $\mathbf{u}$ and taking $\mathbf{w}$ as test function in the resulting equation, we get

$$
\frac{1}{2} \frac{\mathrm{d}}{\mathrm{d} t}|\mathbf{w}|_{2}^{2}+\sum_{i, j=1}^{3} \int_{\Omega}\left[\mathcal{T}_{i j}(\mathbf{S}(\mathbf{u}))-\mathcal{T}_{i j}(\mathbf{S}(\mathbf{v}))\right] S_{i j}(\mathbf{w}) \mathrm{d} \mathbf{x}=-\int_{\Omega}(\mathbf{w} \cdot \nabla) \mathbf{u w} \mathrm{d} \mathbf{x}
$$

Moreover, from the definition of the tensor $\mathcal{T}$ we have

$$
\sum_{i, j=1}^{3} \int_{\Omega}\left[\mathcal{T}_{i j}(\mathbf{S}(\mathbf{u}))-\mathcal{T}_{i j}(\mathbf{S}(\mathbf{v}))\right] S_{i j}(\mathbf{w}) \mathrm{d} \mathbf{x} \geq c_{1} \sum_{i, j=1}^{3} \int_{\Omega}\left|S_{i j}(\mathbf{w})\right|^{2} \mathrm{~d} \mathbf{x}, \quad \text { with } \quad c_{1}>0
$$

Using Korn's inequality

$$
\left(\int_{\Omega}|\mathbf{S}(\mathbf{u})|^{p} \mathrm{~d} \mathbf{x}\right)^{1 / p} \geq C_{p}|\nabla \mathbf{u}|_{p} \text { for } \mathbf{u} \in W_{0}^{1, p} \text { with } C_{p}>0 \quad(1<p<+\infty)
$$

and Hölder's inequality we obtain from (12)

$$
\frac{1}{2} \frac{\mathrm{d}}{\mathrm{d} t}|\mathbf{w}|_{2}^{2}+c_{2}|\nabla \mathbf{w}|_{2}^{2} \leq \int_{\Omega}|\mathbf{w}|^{2}|\nabla \mathbf{u}| \mathrm{d} \mathbf{x} \leq|\nabla \mathbf{u}|_{3}|\mathbf{w}|_{3}^{2}
$$

In three dimensions we have the embedding

$$
H^{1}(\Omega) \subset L^{6}(\Omega)
$$

from which we deduce

$$
|\mathbf{w}|_{3} \leq|\mathbf{w}|_{2}^{1 / 2}|\mathbf{w}|_{6}^{1 / 2} \leq c_{3}|\mathbf{w}|_{2}^{1 / 2}|\nabla \mathbf{w}|_{2}^{1 / 2}
$$

Moreover, it follows from (14), via Young's inequality, that

$$
\frac{\mathrm{d}}{\mathrm{d} t}|\mathbf{w}|_{2}^{2}+c_{4}|\nabla \mathbf{w}|_{2}^{2} \leq c_{5}|\nabla \mathbf{u}|_{3}^{2}|\mathbf{w}|_{2}^{2}
$$

\footnotetext{
${ }^{3}$ This reference can be found at the internet address www-lmc.imag.fr/lmc-edp/Delia. Jiroveanu
} 
Since the function $g(t)=|\nabla \mathbf{u}|_{3}^{2}$ is integrable on $] 0, T[$ and $\mathbf{w}(0)=0$, using Gronwall's inequality we get

$$
|\mathbf{w}(t)|_{2}^{2}=0 \text { on }[0, T]
$$

and thus the uniqueness of the solution to problem $(\mathcal{S})$.

The uniform in time regularity is related to the asymptotic behavior of the solution that we now consider.

\subsection{Asymptotic behavior and regularity}

Let $\mathbf{u}_{0} \in H$ and suppose now that $\mathbf{f} \in L^{2}(\Omega)^{3}$ is time independent.

Thanks to Aubin-Lions Lemma 1.1, the unique weak solution given by Theorem 1.2 is continuous

$$
\mathbf{u} \in \mathcal{C}((0, T) ; H)
$$

Consequently, we can define the family of operators $(S(t))_{t \geq 0}$ by

$$
\begin{aligned}
S(t): H & \rightarrow H \\
\mathbf{u}_{0} & \mapsto S(t) \mathbf{u}_{0}=\mathbf{u}(t) \quad \text { solution to problem }(\mathcal{S}) .
\end{aligned}
$$

It is easy to show that this family form a continuous semigroup for which we have:

Proposition 1.3. There exists a ball

$$
B_{\rho}=\left\{\mathbf{u} \in V ; \quad|\nabla \mathbf{u}|_{3} \leq \rho\right\}
$$

which absorbs all the balls in the space $H$.

Proof. Taking in (11) $\mathbf{u}$ as test function and using the property

$$
\int_{\Omega}(\mathbf{u} \cdot \nabla) \mathbf{u} \cdot \mathbf{u} \mathrm{d} \mathbf{x}=0 \quad \forall \mathbf{u} \in V
$$

we obtain

$$
\frac{\mathrm{d}}{\mathrm{d} t}|\mathbf{u}|_{2}^{2}+\sum_{i, j=1}^{3} \int_{\Omega} \mathcal{T}_{i j}(\mathbf{S}(\mathbf{u})) S_{i j}(\mathbf{u}) \mathrm{d} \mathbf{x}=\langle\mathbf{f}, \mathbf{u}\rangle
$$

The tensor $\mathcal{T}$ can be represented through a nonnegative potential $\theta: \mathbf{R}^{9} \rightarrow \mathbf{R}$ given by

$$
\theta(\mathbf{S})=\int_{0}^{|\mathbf{S}|^{2}}\left(\nu+\nu_{1} \mathbf{y}^{1 / 2}\right) \mathrm{d} \mathbf{y}
$$

Indeed, we have

$$
\mathcal{T}_{i j}(\mathbf{S})=\frac{\partial \theta(\mathbf{S})}{\partial S_{i j}} \quad \forall i, j=1,2,3 .
$$

Moreover,

$$
\theta(0)=0 \quad \text { and } \quad \frac{\partial \theta(0)}{\partial S_{i j}}=0 \quad \forall i, j=1,2,3
$$


It follows from (18) that

$$
\mathcal{T}_{i j} S_{i j}=2\left(\nu+\nu_{1}|\mathbf{S}|\right)|\mathbf{S}|^{2} \geq c_{1}(1+|\mathbf{S}|)|\mathbf{S}|^{2}
$$

and thus we find

$$
\frac{1}{2} \frac{\mathrm{d}}{\mathrm{d} t}|\mathbf{u}|_{2}^{2}+\nu c_{1} C_{2}^{2}|\nabla \mathbf{u}|_{2}^{2}+\nu_{1} c_{1} C_{3}^{3}|\nabla \mathbf{u}|_{3}^{3} \leq\langle\mathbf{f}, \mathbf{u}\rangle
$$

Applying Hölder's inequality, followed by Poincaré's inequality

$$
|\mathbf{u}|_{2} \leq \lambda_{1}^{-1 / 2}|\nabla \mathbf{u}|_{2}^{1}
$$

where $\lambda_{1}$ is the first eigenvalue of the Stokes operator, and using the following inequality

$$
\lambda_{1}{ }^{-1 / 2}|\mathbf{f}|_{2}|\nabla \mathbf{u}|_{2} \leq \frac{\nu c_{1} C_{2}^{2}}{2}|\nabla \mathbf{u}|_{2}^{2}+\frac{1}{2 \nu c_{1} C_{2}^{2} \lambda_{1}}|\mathbf{f}|_{2}^{2}
$$

we obtain

$$
\frac{\mathrm{d}}{\mathrm{d} t}|\mathbf{u}|_{2}^{2}+\nu c_{1} C_{2}^{2}|\nabla \mathbf{u}|_{2}^{2}+2 \nu_{1} c_{1} C_{3}^{3}|\nabla \mathbf{u}|_{3}^{3} \leq \frac{|\mathbf{f}|_{2}^{2}}{\nu c_{1} C_{2}^{2} \lambda_{1}}
$$

respectively,

$$
\frac{\mathrm{d}}{\mathrm{d} t}|\mathbf{u}|_{2}^{2}+\nu \lambda_{1} c_{1} C_{2}^{2}|\mathbf{u}|_{2}^{2}+2 \nu_{1} c_{1} C_{3}^{3}|\nabla \mathbf{u}|_{3}^{3} \leq \frac{|\mathbf{f}|_{2}^{2}}{\nu c_{1} C_{2}^{2} \lambda_{1}}
$$

The classical Gronwall lemma gives

$$
|\mathbf{u}|_{2}^{2} \leq\left|\mathbf{u}_{0}\right|_{2}^{2} \exp \left(-\nu \lambda_{1} c_{1} C_{2}^{2} t\right)+\frac{|\mathbf{f}|_{2}^{2}}{\nu^{2} c_{1}^{2} C_{2}^{4} \lambda_{1}^{2}}\left(1-\exp \left(-\nu \lambda_{1} c_{1} C_{2}^{2} t\right)\right)
$$

and thus we have

$$
\limsup _{t \rightarrow \infty}|\mathbf{u}|_{2} \leq \rho_{0}, \quad \text { with } \rho_{0}=\frac{|\mathbf{f}|_{2}}{\nu c_{1} C_{2}^{2} \lambda_{1}}
$$

From (22) we infer that the balls of $H$ of radius $\rho$ are absorbing for all $\rho>\rho_{0}$.

Indeed, let $\rho>\rho_{0}$ and denote by $B_{0}$ the ball $B_{H}(0, \rho)$. Let $B$ be any bounded set in $H$. Then, there exists $R>0$ such that $B \subset B(0, R)$. Hence we have

$$
|\mathbf{u}(t)|^{2} \leq R^{2} \exp \left(-\nu \lambda_{1} c_{1} C_{2}^{2} t\right)+\rho_{0}^{2}\left(1-\exp \left(-\nu \lambda_{1} c_{1} C_{2}^{2} t\right)\right)
$$

It is obvious that the condition

$$
R^{2} \exp \left(-\nu \lambda_{1} c_{1} C_{2}^{2} t\right)+\rho^{2}\left(1-\exp \left(-\nu \lambda_{1} c_{1} C_{2}^{2} t\right)\right)<\rho^{2}
$$


implies

$$
S(t) B \subset B_{0} \quad \forall t>t_{0}(B, \rho)=t_{0}=\frac{1}{\nu \lambda_{1} c_{1} C_{2}^{2}} \log \frac{R^{2}}{\rho^{2}-\rho_{0}^{2}},
$$

which proves that $B_{0}$ is an absorbing set in $H$.

If in addition we integrate the equation (21) between $t$ and $t+1$, for $t \geq t_{0}$, we obtain the following estimates

$$
\begin{aligned}
2 \nu_{1} C_{3}^{3} \int_{t}^{t+1}|\nabla \mathbf{u}(s)|_{3}^{3} \mathrm{~d} s & \leq \frac{|\mathbf{f}|_{2}^{2}}{\nu \lambda_{1} c_{1} C_{2}^{2}}+|\mathbf{u}(t)|_{2}^{2} \\
& \leq \frac{|\mathbf{f}|_{2}^{2}}{\nu \lambda_{1} c_{1} C_{2}^{2}}+\rho_{0}^{2}=C_{0}\left(\rho_{0}\right) .
\end{aligned}
$$

We note $C(\rho)=\frac{C_{0}\left(\rho_{0}\right)}{2 \nu_{1} c_{1} C_{3}^{3}}$.

Taking now $\frac{\mathrm{d} \mathbf{u}}{\mathrm{d} t}$ as test function in the weak formulation $(11)$ of problem $(\mathcal{S})$ then applying Hölder and Young inequalities, we get

$$
\frac{1}{2}\left|\frac{\mathrm{d} \mathbf{u}}{\mathrm{d} t}\right|_{2}^{2}+\frac{\mathrm{d}}{\mathrm{d} t}|\theta(\mathbf{S})|_{1} \leq C\left(|\mathbf{f}|_{2}^{2}+|\nabla \mathbf{u}|_{3}^{2}|\mathbf{u}|_{6}^{2}\right)
$$

Since, by the Gagliardo-Nirenberg inequality,

$$
|\mathbf{u}|_{6} \leq K_{1}|\nabla \mathbf{u}|_{3}^{2 / 3}|\mathbf{u}|_{2}^{1 / 3}
$$

and

$$
K_{2}|\nabla \mathbf{u}|_{3}^{3} \leq|\theta(\mathbf{S})|_{1} \leq K_{3}\left(1+|\nabla \mathbf{u}|_{3}^{3}\right)
$$

the term $|\nabla \mathbf{u}|_{3}^{2}|\mathbf{u}|_{6}^{2}$ can be estimated by

$$
|\nabla \mathbf{u}|_{3}^{2}|\mathbf{u}|_{6}^{2} \leq K_{1}|\mathbf{u}|_{2}^{2 / 3}|\nabla \mathbf{u}|_{3}^{10 / 3} \leq K_{4}|\mathbf{u}|_{2}^{2 / 3}|\nabla \mathbf{u}|_{3}^{1 / 3}|\theta(\mathbf{S})|_{1} .
$$

Thus we obtain the following inequality

$$
\frac{\mathrm{d}}{\mathrm{d} t}|\theta(\mathbf{S})|_{1} \leq|\mathbf{f}|_{2}^{2}+K_{4}|\mathbf{u}|_{2}^{2 / 3}|\nabla \mathbf{u}|_{3}^{1 / 3}|\theta(\mathbf{S})|_{1} .
$$

Let

$$
y=|\theta(\mathbf{S})|_{1}, \quad g=K_{4}|\mathbf{u}|_{2}^{2 / 3}|\nabla \mathbf{u}|_{3}^{1 / 3}, \quad h=|\mathbf{f}|_{2}^{2}
$$

thus for all $t \geq t_{0}$ we have

$$
\begin{aligned}
\int_{t}^{t+1} y(s) \mathrm{d} s & \leq K_{3}(1+C(\rho)):=\alpha_{3} \\
\int_{t}^{t+1} g(s) \mathrm{d} s & \leq \rho^{2 / 3} \int_{t}^{t+1}|\nabla \mathbf{u}|_{3}^{1 / 3} d s \stackrel{(26)}{\leq} K_{5} \rho^{2 / 3} C(\rho):=\alpha_{1} \\
|\mathbf{f}|_{2}^{2} & :=\alpha_{2} .
\end{aligned}
$$


And, thanks to the uniform Gronwall lemma (see [47], p. 91) we obtain

$$
|\theta(\mathbf{S})|_{1} \leq\left(\alpha_{3}+\alpha_{2}\right) \exp \alpha_{1} \quad \text { for all } t \geq t_{0}+1
$$

Using (29) we now get

$$
|\nabla \mathbf{u}|_{3}^{3} \leq K\left(\alpha_{3}+\alpha_{2}\right) \exp \alpha_{1}:=\alpha^{3}
$$

Since $\alpha=\alpha(\rho)$ it is straightforward to verify that the balls $B_{\rho}=\left\{\mathbf{u} \in V ;|\nabla \mathbf{u}|_{3} \leq \rho\right\}$ absorb all bounded sets of $H$ if $\rho>\alpha$.

As a consequence of Proposition 1.3 we have:

Theorem 1.4. The problem $(\mathcal{S})$ has a global attractor

$$
\mathcal{A}=\cap_{s>0}\left(\cup_{t \geq s} S(t) B_{\rho}\right) .
$$

Remark 1.5. Following Malek \& Necas [36], we employed in [25] the method of short trajectories in order to prove that the attractor (36) has a finite fractal dimension.

\section{Vorticity EQUATIONS, SINGULARITY CONDITIONS AND IMPROVED SGS MODELS}

For a better understanding of turbulence mechanisms, it is useful to consider the velocity-vorticity formulation of the Navier-Stokes equations, because it brings more to the fore the difference between the two and the three dimensional cases. Taking the curl of the Navier-Stokes equations yields

$$
\partial_{t} \boldsymbol{\omega}+(\mathbf{u} \cdot \nabla) \boldsymbol{\omega}=(\boldsymbol{\omega} \cdot \nabla) \mathbf{u}+\nu \Delta \boldsymbol{\omega}
$$

where $\boldsymbol{\omega}=\nabla \times \mathbf{u}$ is the vorticity. This describes the generation and the transport of vorticity for a general three dimensional flow. The last term in the right hand side expresses the effects of viscous diffusion on the vorticity distribution. In three dimensional flows at high Reynolds numbers, the viscous diffusion of vorticity is dominated by the vortex stretching, represented in $(37)$ by the term $(\boldsymbol{\omega} \cdot \nabla) \mathbf{u}$. It is argued to be the most important mechanism in the turbulence dynamics. By this mechanism the turbulent energy is transferred from larger to smaller scales. The stretching term is responsible in this case of the intensification and the reorientation of the vorticity and it may contribute to the production of finite time singularities. In a two dimensional flow, the vorticity merely behaves as a passive scalar that follows fluid particle paths (for a turbulent flow, the influence of the viscosity is small). In this case, the vorticity vector is confined to a plane perpendicular to the flow and its magnitude is uniformly bounded.

If $\boldsymbol{\omega}$ is divergence-free, it is possible to recover the velocity from the vorticity field through the stream-vector $\Psi$ that satisfies $-\nabla^{2} \Psi=\boldsymbol{\omega}$, by taking $\mathbf{u}=\nabla \times \Psi$. Thus, one obtains the following formula for the velocity field, known in the literature as the Biot-Savart low [6]

$$
\mathbf{u}(\mathbf{x})=-\frac{1}{4 \pi} \int_{\Omega} \frac{\left(\mathbf{x}-\mathbf{x}^{\prime}\right) \times \omega\left(\mathbf{x}^{\prime}\right)}{\left|\mathbf{x}-\mathbf{x}^{\prime}\right|^{3}} \mathrm{~d} \mathbf{x}^{\prime} .
$$

A similar approach to the grid-based LES in primitive variables can be obtained for the velocity-vorticity formulation. Filtering the vorticity transport equations (37) we get

$$
\partial_{t} \bar{\omega}_{i}+\bar{u}_{j} \cdot \partial_{j} \bar{\omega}_{i}=\bar{\omega}_{j} \cdot \partial_{j} \bar{u}_{i}+\nu \nabla^{2} \bar{\omega}_{i}-\partial_{j} T_{i j}
$$

where $T_{i j}=\left(\overline{\omega_{i} u_{j}}-\bar{\omega}_{i} \bar{u}_{j}\right)-\left(\overline{u_{i} \omega_{j}}-\bar{u}_{i} \bar{\omega}_{j}\right)$ is the subgrid scale vorticity stress, which accounts for the effect of unresolved velocity and vorticity fluctuations. As for the filtered Navier-Stokes equations in primitive variables, 
one must provide a model for the vorticity stress $T$ in order to close the filtered vorticity transport equation (38). A natural closure model for this problem is the vorticity version of the Smagorinsky model given by

$$
T=\nu_{t} \nabla \boldsymbol{\omega} \quad \text { with } \quad \nu_{t}=\left(C_{\mathrm{s}} \Delta\right)^{2}|\boldsymbol{\omega}| .
$$

As we have already mentioned, the Smagorinsky model is too dissipative. We investigate here two selective SGS models which avoid to some extent this difficulty. These models can be understood by looking at the mechanisms of vorticity blow-up in the Navier-Stokes equations.

\subsection{Anisotropic selective model}

We start here from the gradient model [48]

$$
\tau_{i j} \simeq\left(C_{\mathrm{a}} \Delta\right)^{2} \partial_{k} u_{i} \partial_{k} u_{j}
$$

where $\Delta$ is the filter width. As already mentioned this model, although satisfactory from the point of view of correlations with DNS or experimental results, has the drawback of producing backward transfer of energy which ultimately makes its implementation unstable.

To understand the links between the gradient model and the problem of regularity in the Navier-Stokes equations, let us consider the enstrophy budget resulting in the Navier-Stokes equations. Multiplying (37) by $\boldsymbol{\omega}$, due to the fact that $\mathbf{u}$ is divergence free, one obtains

$$
\frac{\mathrm{d}}{\mathrm{d} t}|\boldsymbol{\omega}|^{2}=\int_{\Omega}(\boldsymbol{\omega} \cdot \nabla \mathbf{u}) \cdot \boldsymbol{\omega} \mathrm{d} \mathbf{x}-\nu \int_{\Omega}|\nabla \boldsymbol{\omega}|^{2} \mathrm{~d} \mathbf{x} .
$$

The contribution of the stretching term can be estimated as follows. If we denote by $\mathbf{S}^{+}$the positive part of the - symmetric - stress tensor $\mathbf{S}$ (we recall that $\left.S_{i j}=1 / 2\left(\partial_{i} u_{j}+\partial_{j} u_{i}\right)\right)$ we have

$$
\int_{\Omega}(\boldsymbol{\omega} \cdot \nabla \mathbf{u}) \cdot \boldsymbol{\omega} \mathrm{d} \mathbf{x}=\int_{\Omega} \omega_{i} S_{i j} \omega_{j} \mathrm{~d} \mathbf{x} \leq \int_{\Omega} \omega_{i} S_{i j}^{+} \omega_{j} \mathrm{~d} \mathbf{x} .
$$

As a result, enstrophy may increase, with possible vorticity blow-up, when the vorticity is aligned with the directions corresponding to positive eigenvalues of $\mathbf{S}$. If one wishes to limit the enstrophy increase in order to maintain smooth solutions, this inequality suggests an eddy viscosity tensor with strength proportional to $S^{+}$. We next observe that for an incompressible flow the trace of $\mathbf{S}$ vanishes and its eigenvalues thus sum to zero. There are therefore two natural candidates: $\mathbf{S}^{+}$itself and $-\mathbf{S}^{-}$.

A look at a typical configuration where enstrophy increases, as sketched in Figure 1, eliminates the choice of $\mathbf{S}^{+}$: it corresponds to two directions with negative $\mathbf{S}$ eigenvalues (for clarity only one is shown) and one, with positive eigenvalue, locally parallel to the vorticity (a bathtube type configuration). In that case, vorticity exhibits strong gradients along the first two directions, which are also the directions along which small scales are produced, while it is nearly constant along the third direction (this follows from the fact that vorticity is divergence free). If one keeps in mind that the goal of subgrid-scale models is to dissipate enstrophy in the directions of production of small scales, this leads to the choice

$$
\nu_{t}=-\left(C_{\mathrm{a}} \Delta\right)^{2} \mathbf{S}^{-} .
$$

Going back to the velocity formulation, this model can also be interpreted as a selective version of the gradient model (39), where only dissipation directions are kept in the turbulent viscosity tensor, avoiding the numerically unstable backward transfer of energy. In $[14]^{4}$ an implementation procedure of this model was derived in order

\footnotetext{
${ }^{4}$ References $[14,17]$ can be found at the internet address www-lmc.imag.fr/lmc-edp/Georges-Henri.Cottet
} 

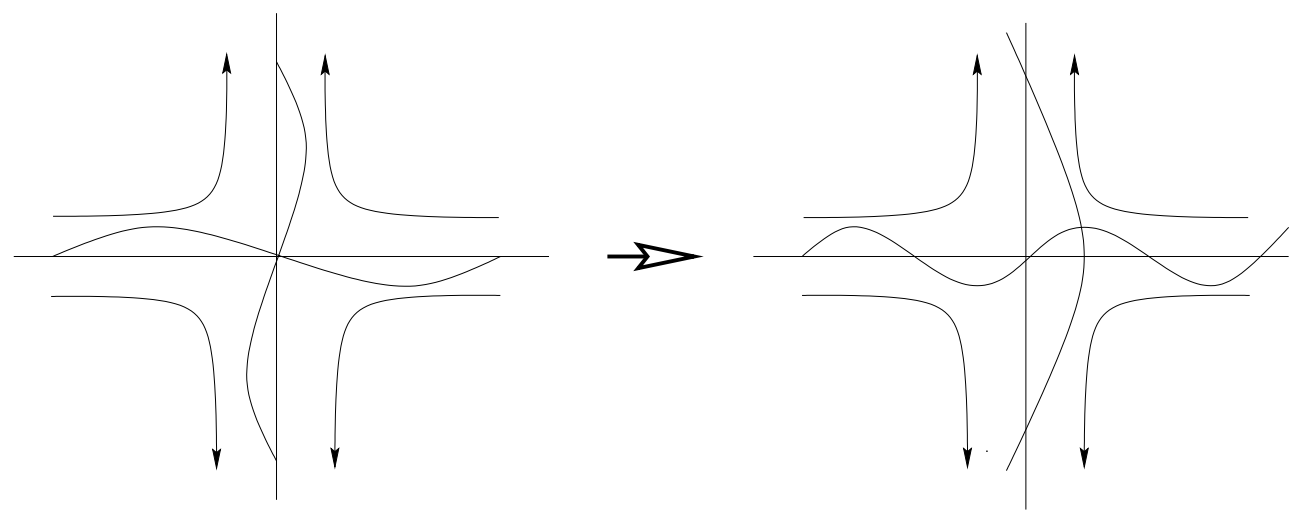

FiguRE 1. Flow around an hyperbolic point and production of small or large scale along the strain directions.

to avoid the diagonalization of $\mathbf{S}$ at every grid point. For a sake of completeness we reproduce here the main steps of this derivation.

It uses a numerical filter function $\zeta$ satisfying the following moment conditions

$$
\int x_{k} x_{l} \zeta(\mathbf{x}) \mathrm{d} \mathbf{x}=\delta_{k l}, \quad k, l=1,2,3
$$

where $x_{k}$ denote the components of $\mathbf{x}$. Such a filter can be easily constructed by proper rescaling of any positive function satisfying symmetry properties (e.g. functions with spherical symmetry, or functions constructed through tensor product of one-dimensional even functions).

Starting from (39), we first write

$$
\Delta^{2} \partial_{k} u_{i}(\mathbf{x}) \partial_{k} u_{j}(\mathbf{x})=\Delta^{-3} \int \partial_{k} u_{i}(\mathbf{x}) \partial_{l} u_{j}(\mathbf{x})\left(y_{k}-x_{k}\right)\left(y_{l}-x_{l}\right) \zeta\left(\frac{\mathbf{y}-\mathbf{x}}{\Delta}\right) \mathrm{d} \mathbf{y}
$$

where we recall that the summation of repeated indices is implied. Taylor expansions of $u_{i}$ and $u_{j}$ around $\mathbf{x}$, yield

$$
\Delta^{2} \partial_{k} u_{i}(\mathbf{x}) \partial_{k} u_{j}(\mathbf{x})=\Delta^{-3}\left\{\int\left[u_{j}(\mathbf{y})-u_{j}(\mathbf{x})\right]\left[u_{i}(\mathbf{y})-u_{i}(\mathbf{x})\right] \zeta\left(\frac{\mathbf{y}-\mathbf{x}}{\Delta}\right) \mathrm{d} \mathbf{y}+O\left(\Delta^{2}\right)\right\}
$$

We then take the divergence of (43) to obtain

$$
\partial_{j}\left[\partial_{k} u_{i}(\mathbf{x}) \partial_{k} u_{j}(\mathbf{x})\right] \simeq A_{i}+B_{i}
$$

after cancellation of the term involving the divergence of $\mathbf{u}$, where

$$
\begin{gathered}
A_{i}=-\Delta^{-3} \int\left[u_{j}(\mathbf{y})-u_{j}(\mathbf{x})\right] \partial_{j} u_{i}(\mathbf{x}) \zeta\left(\frac{\mathbf{y}-\mathbf{x}}{\Delta}\right) \mathrm{d} y \\
B_{i}=-\Delta^{-4} \int\left[u_{j}(\mathbf{y})-u_{j}(\mathbf{x})\right]\left[u_{i}(\mathbf{y})-u_{i}(\mathbf{x})\right] \partial_{j} \zeta\left(\frac{\mathbf{y}-\mathbf{x}}{\Delta}\right) \mathrm{d} \mathbf{y} .
\end{gathered}
$$

It is readily seen that $A_{i}$ is a convective term: if one sets $\lambda=\int \zeta(\mathbf{y}) \mathrm{d} \mathbf{y}$ and

$$
\hat{\mathbf{u}}(\mathbf{x})=\frac{1}{\lambda \Delta^{3}} \int \mathbf{u}(\mathbf{y}) \zeta\left(\frac{\mathbf{y}-\mathbf{x}}{\Delta}\right) \mathrm{d} \mathbf{y}
$$


then $A_{i}$ can be rewritten as $(\hat{\mathbf{u}}-\mathbf{u}) \nabla u_{i}$. It thus does not contribute to the energy balance. Since we wish to model the transfer of energy between large and small scales, we will only be interested in $B_{i}$. We end up with the following form of the gradient model

$$
\partial_{j} \tau_{i j} \simeq-C_{\mathrm{a}}^{2} \Delta^{-4} \int\left[u_{j}(\mathbf{y})-u_{j}(\mathbf{x})\right]\left[u_{i}(\mathbf{y})-u_{i}(\mathbf{x})\right] \partial_{j} \zeta\left(\frac{\mathbf{y}-\mathbf{x}}{\Delta}\right) \mathrm{d} \mathbf{y} .
$$

To identify the positive contribution of this model to energy dissipation, we multiply this quantity by $\mathbf{u}$ and integrate over $\Omega$. Writing $\mathbf{u}(\mathbf{x})=\frac{1}{2}[\mathbf{u}(\mathbf{x})+\mathbf{u}(\mathbf{y})]+\frac{1}{2}[\mathbf{u}(\mathbf{x})-\mathbf{u}(\mathbf{y})]$ and using the symmetry of $\zeta$, we are left with

$$
\mathcal{D}=\frac{C_{\mathrm{a}}^{2}}{2} \Delta^{-4} \int[\mathbf{u}(\mathbf{y})-\mathbf{u}(\mathbf{x})] \cdot \nabla \zeta\left(\frac{\mathbf{y}-\mathbf{x}}{\Delta}\right)|\mathbf{u}(\mathbf{y})-\mathbf{u}(\mathbf{x})|^{2} \mathrm{~d} \mathbf{x} \mathrm{d} \mathbf{y} .
$$

This formula gives a way to measure the local dissipation at a given point $\mathbf{x}$ as well, by restricting the integral over y only. It also enables us to formulate a strictly dissipative model. This model reads

$$
\left.\partial_{j} \tau_{i j}(\mathbf{x}) \simeq C_{\mathrm{a}}^{2} \Delta^{-4} \int\left\{[\mathbf{u}(\mathbf{x})-\mathbf{u}(\mathbf{y})] \cdot \nabla \zeta\left(\frac{\mathbf{x}-\mathbf{y}}{\Delta}\right)\right]\right\}_{+}[\mathbf{u}(\mathbf{x})-\mathbf{u}(\mathbf{y})] \mathrm{d} \mathbf{y}
$$

where $a_{+}=\max (0, a)$. Unlike the traditional clipping strategies, which would consist in replacing $\partial_{j} \tau_{i j}$ by zero whenever the global energy budget would have the wrong sign, this technique respects the anisotropy of the original gradient model, in the sense that it allows to dissipate in one or more directions while controlling the backscatter which would arise in the other directions. Typically, $\zeta$ is a decreasing function of the radius, and the turbulent viscosity tensor given by this model dissipates energy between points which are compressed by the flow. As it is proved in [14], this model has also the correct asymptotic behavior near boundaries.

\subsection{Smagorinsky selective model}

The aim of the model proposed here is to reduce the support of the eddy-viscosity of the Smagorinsky model in order to correct its excessive dissipation. This model is reminiscent to a selective model proposed by David [18] in the context of the so-called structure-function model of Metais and Lesieur [39]. The idea is to apply a turbulence model only in regions of intense vortex activity and where the flow is strongly three-dimensional. This can be done by multiplying the eddy viscosity with a filter function acting like a detector of turbulence zones. This filter is based on a comparisons of the variation of the vorticity direction with a given threshold. In a mathematical approach of LES, this criterion can be understood in the light of a sharp result of P. Constantin and C. Fefferman giving necessary conditions for the loss of regularity in the Navier-Stokes equations.

This result, which is a refinement of a well-known result of Beale, Kato and Majda [2], can be stated as follows [11]: Assume that there exist positive constants $\Omega$ and $\rho$ such that, if the vorticity magnitude at two locations $\mathbf{x}$ and $\mathbf{x}^{\prime}$ exceeds $\Omega$ then

$$
\sin \beta\left(\mathbf{x}, \mathbf{x}^{\prime}, t\right) \leq \frac{\left|\mathbf{x}-\mathbf{x}^{\prime}\right|}{\rho},
$$

where $\beta\left(\mathbf{x}, \mathbf{x}^{\prime}, t\right)$ is the angle between the vorticity vectors at locations $\mathbf{x}$ and $\mathbf{x}^{\prime}$ at time $t$. Then the Navier-Stokes equation have a strong solution (that is with bounded vorticity) on $[0, T]$.

Note that this result has recently been further improved [3] to allow for an Holderian instead of Lipschitz control of the angle. To illustrate this result, let us consider the flow corresponding to the reconnection of two vortex tubes. Vortex reconnection is a classical situation where vorticity blow-up is suspected, at least in the inviscid case. It occurs for instance when two vortex tubes approach each other and collide to form vortex rings which ultimately separate. Figure 2 shows isosurfaces of vorticity magnitude around the reconnection time at a Reynolds number of 3500 , together with a few filaments. One can observe that filaments, which initially were almost parallel straight lines, undergo rapid change of orientations in the core of the vortices. 


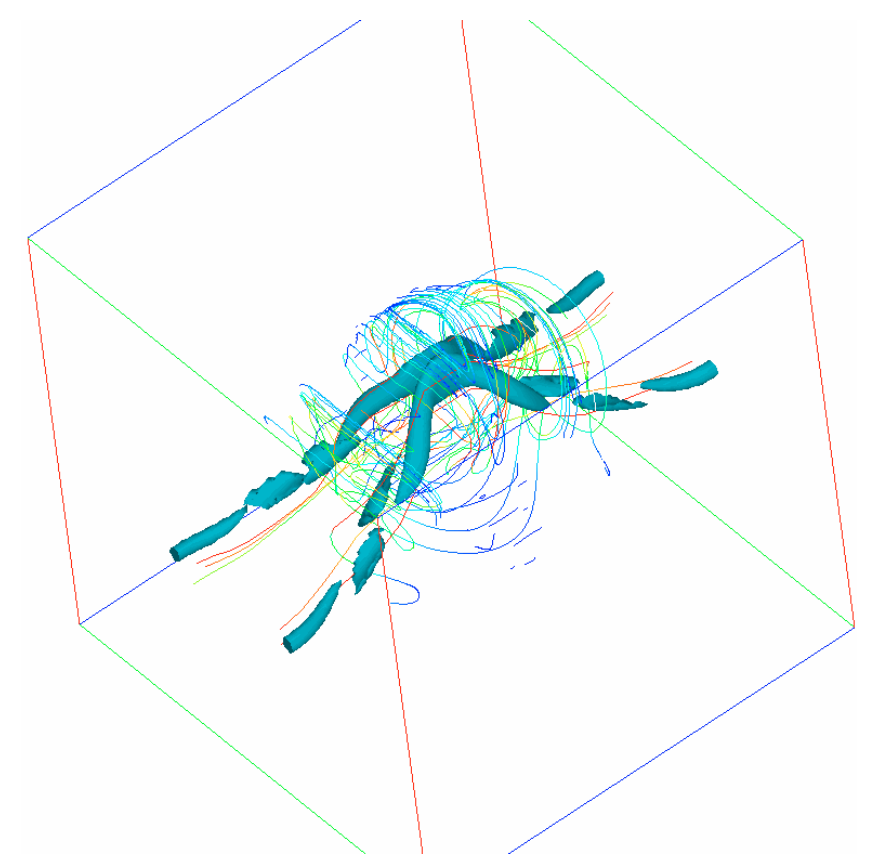

FIGURE 2. Vortex reconnection of two initially almost parallel vortex tubes. Isosurfaces of vorticity magnitude and filaments of vorticity.

If one believes that SGS models must maintain smooth solutions with minimal dissipation, turbulent viscosity should only be active where vorticity is strong and changes rapidly its direction.

In practice, the criterion used for detecting such regions is the following: we compute the angle between the vorticity at a given grid point and the average vorticity at the six closest neighboring points defined by

$$
\beta_{m}(\mathbf{x}, t)=\arcsin \frac{\boldsymbol{\omega}(\mathbf{x}, t) \times \boldsymbol{\omega}_{m}(\mathbf{x}, t)}{|\boldsymbol{\omega}(\mathbf{x}, t)| \cdot\left|\boldsymbol{\omega}_{m}(\mathbf{x}, t)\right|}
$$

where $\boldsymbol{\omega}_{m}(\mathbf{x}, t)$ is given by the integral

$$
\boldsymbol{\omega}_{m}(\mathbf{x}, t)=\frac{1}{\int_{|\mathbf{r}|=\Delta \mathbf{x}} \mathrm{d} \mathbf{r}} \int_{|\mathbf{r}|=\Delta \mathbf{x}} \boldsymbol{\omega}(\mathbf{x}+\mathbf{r}, t) \mathrm{d} \mathbf{r}
$$

evaluated in the discrete space by the average

$$
\boldsymbol{\omega}_{m}(\mathbf{x}, t)=\frac{1}{6} \sum_{i=1}^{3}\left(\boldsymbol{\omega}\left(\mathbf{x}+\Delta_{i} x_{i}, t\right)+\boldsymbol{\omega}\left(\mathbf{x}-\Delta_{i} x_{i}, t\right)\right) .
$$

Next, we define the function filter

$$
\Psi(\mathbf{x}, t)= \begin{cases}1, & \text { if } \beta_{0} \leq \beta_{m} \leq \pi-\beta_{0} \\ 0, & \text { otherwise }\end{cases}
$$

where $\beta_{0}$ is some threshold angle. The value picked for $\beta_{0}$ in practical simulation will be discussed in the next section. 


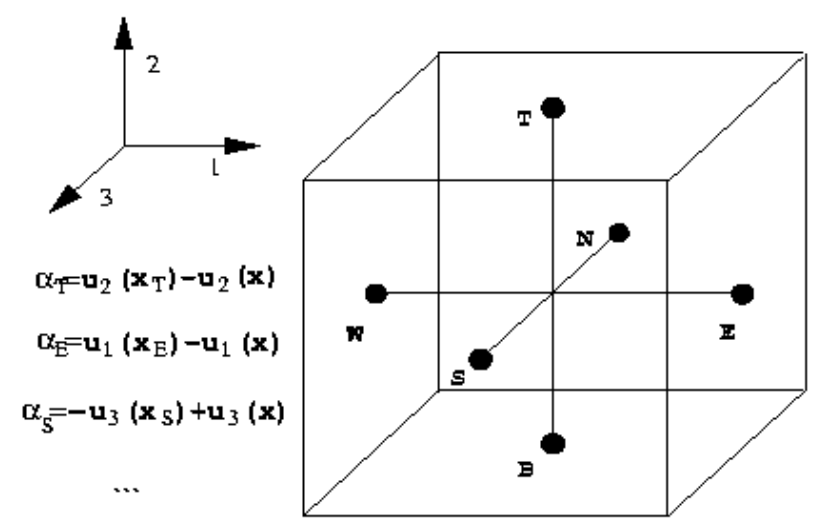

FiguRE 3. Finite-difference stencil of the subgrid-scale model in the case of a uniform grid with a radial hat filter. $\alpha_{T} / \Delta, \alpha_{E} / \Delta, \cdots$ denote the weights of the corresponding grid points (from [14]).

From a physical point of view, the values of $\beta_{m}$ which verify the assumption (47) correspond to a local alignment or antialignment of the vorticity (as in laminar or transition flows); those which do not verify it, indicate a variability of the vortex direction, and thus will correspond to turbulence regions.

Finally, the eddy viscosity for the selective Smagorinsky model is expressed by

$$
\nu_{t}=\Psi(\mathbf{x}, t) \nu_{t}^{\mathrm{sm}} .
$$

\section{NumERICAL RESULTS}

We have chosen as validation tests a case of decaying isotropic turbulence. The incompressible time-decaying flow considered here is similar to those of the grid-turbulence experiments of Comte-Bellot [8]. The reference simulation for our comparison tests is the DNS using $N=512$ Fourier mode in each direction performed by A. Wray and reported in [1] (referred to in the sequel as the reference DNS).

As it is customary, the LES runs used as initial condition the data on a $32^{3}$ grid obtained by a sharp truncation in the Fourier space of the reference DNS. To remain in accordance with the experiment of Comte-Bellot, we followed the usual convention of an initial time of 0.961 .

All computations (DNS as well as LES) were performed with a spectral code in a periodic box and with a Taylor-Reynolds number $R e_{\lambda}=104.5$. To eliminate the aliasing errors we used the classical two-third rule [5]. The subgrid-scale models were implemented in the physical space on a uniform grid, with velocity values obtained through FFT. For the numerical filter $\zeta$ which appears in the expression of the selective anisotropic model we have considered a radial function $\zeta(\mathbf{x})=\alpha f(|\mathbf{x}|)$, where $f$ is the classical hat function. As a result, the stencil corresponding to the implementation of (46) involves 7 points, with weights $\alpha$ 's defined from the velocity components as sketched in Figure 3.

In order to demonstrate the need of LES modeling we first performed an under-resolved DNS with $N=32$ Fourier modes. From Figure 4 (left picture) it is clear that at such low resolution the DNS is unable to correctly predict the decay of the kinetic energy: the lack of resolution induces an unphysical accumulation of energy in the tail of the spectra. This can be seen on the right picture of Figure 4 which represents the energy spectra at three different times of the simulations $(1.28,2.45$ and 5.46).

We then tested the ability of the SGS models to match the time evolution of the filtered kinetic energy (the energy carried in the DNS reference calculation by the first 32 modes).

We performed LES simulations with $N=32^{3}$ modes using respectively the classical Smagorinsky model, the selective anisotropic model (46), and the selective version of the Smagorinsky model (50). 

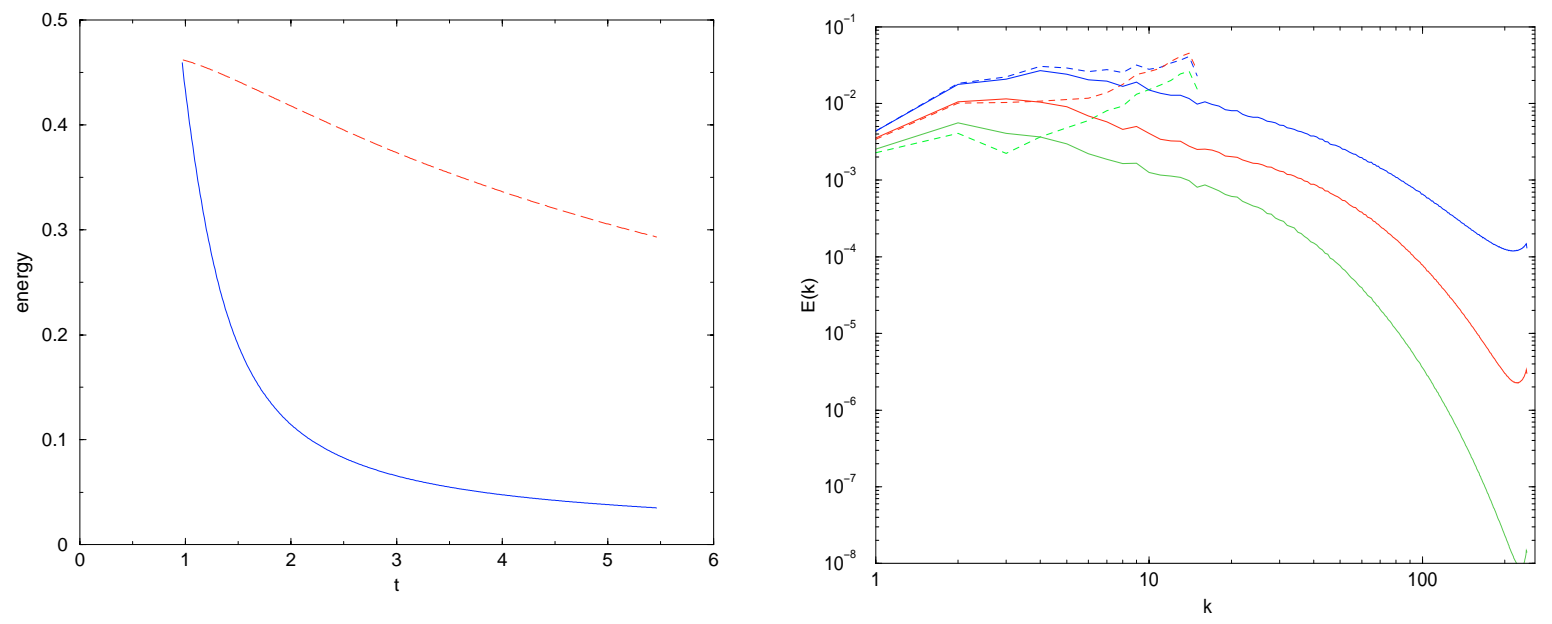

Figure 4. Energy decay (left picture) and energy spectrum at times $t=1.28,2.45$ and 5.46 (right picture) for filtered reference DNS (energy carried by the first 32 modes) (solid) and a DNS with $N=32$ modes (dashed).

In all cases, the velocity-pressure formulation of the Navier-Stokes equations was used, except for the selective Smagorinsky model where the vorticity formulation was chosen. The SGS models were implemented with constants chosen such that they could yield an energy decay which matches the reference DNS data: for the Smagorinsky model we found $C_{\mathrm{s}}=0.27$ and for the anisotropic model $C_{\mathrm{a}}=0.4$. Concerning the selective Smagorinsky model, the threshold angle is another parameter that need to be fixed. Following [18] our criterion was based on the statistical distribution of the angles in homogeneous turbulent flow. However, we observed that, for a given flow, this distribution depends on the resolution. Figure 5 shows the distributions for the initial field sampled on 128 and 32 modes. The peak angle is smaller for the low resolution field, which is not surprising given that this field does not contain small structures. On the basis of this observation we have run the selective Smagorinsky models for the angle values of 5 and 15 degrees (note that [18] uses a value of $20^{\circ}$ ). The corresponding constants were found to be respectively $C_{\mathrm{s}}=0.36$ and $C_{\mathrm{s}}=0.29$. Figure 6 shows the energy decay corresponding to these various cases in comparison with the reference energy obtained from the high resolution DNS.

Once the correct constants have been identified, the difficulty is to accurately recover the decay of the energy spectra in the high modes. The figures which follow present the energy spectra at the three different times already considered.

Figure 7 is a comparison of the Smagorinsky and anisotropic models. It shows that at $32^{3}$ resolution the classical Smagorinsky model is too dissipative in the small scales and that the selective anisotropic models removes to a large extent, in particular for the 2 first times, this excess dissipation. This result confirms those of [17] obtained on the velocity-pressure formulation.

Figure 8 leads to a similar conclusion concerning the selective Smagorinsky model with a threshold angle $\beta_{0}=15^{\circ}$.

To compare more closely the different models, we have plotted in Figure 9 the energy spectra at the latest time (5.46) for all 4 models: Smagorinsky, anisotropic, and selective with the angles $\beta_{0}=5^{\circ}$ and $\beta_{0}=15^{\circ}$. All selective models perform about the same way, with a slight advantage for the anisotropic model. Another observation is that the efficiency of the selective Smagorinsky model does not depend on the choice of the threshold angle (a smaller angle goes together with a smaller model coefficient, as could be expected). For all models, one can still see an excess dissipation in the high modes, compensated by an over production of energy in the low modes. 

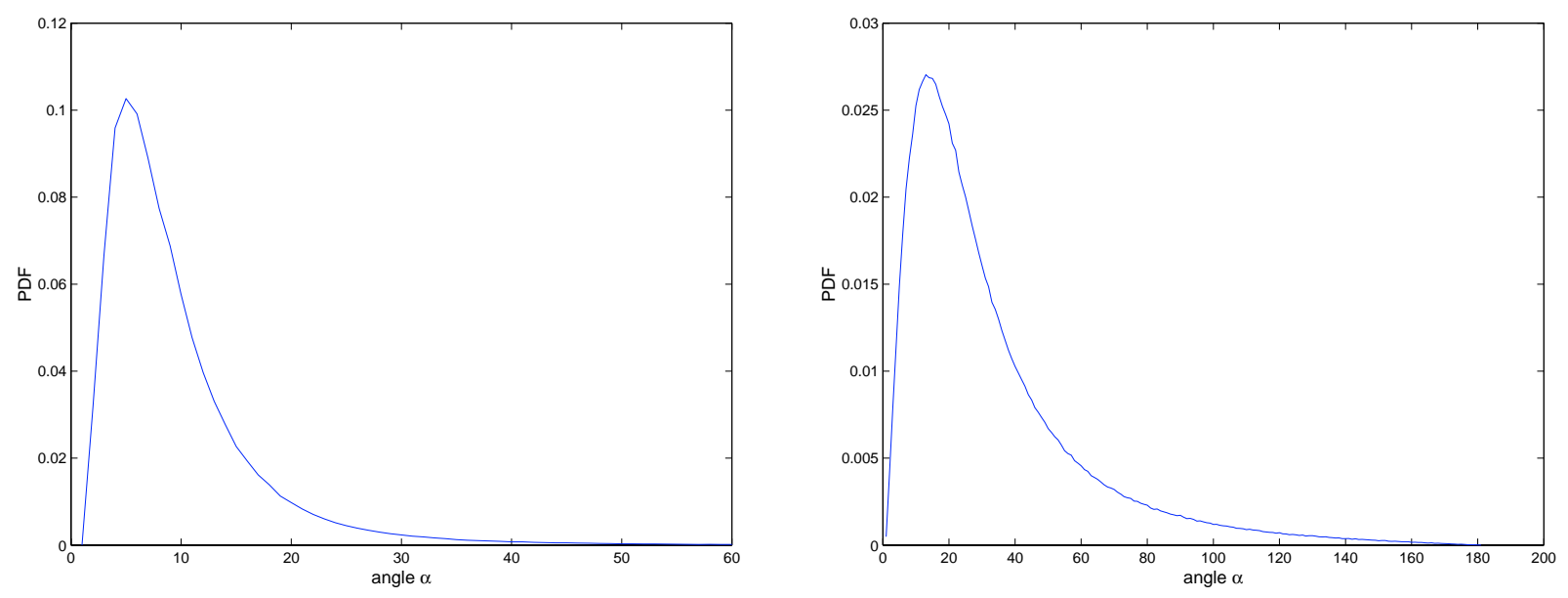

Figure 5. Probability density function for the angles $\beta_{m}$ computed from the initial turbulent field sampled on $N=32$ (left) and $N=128$ (right) modes.

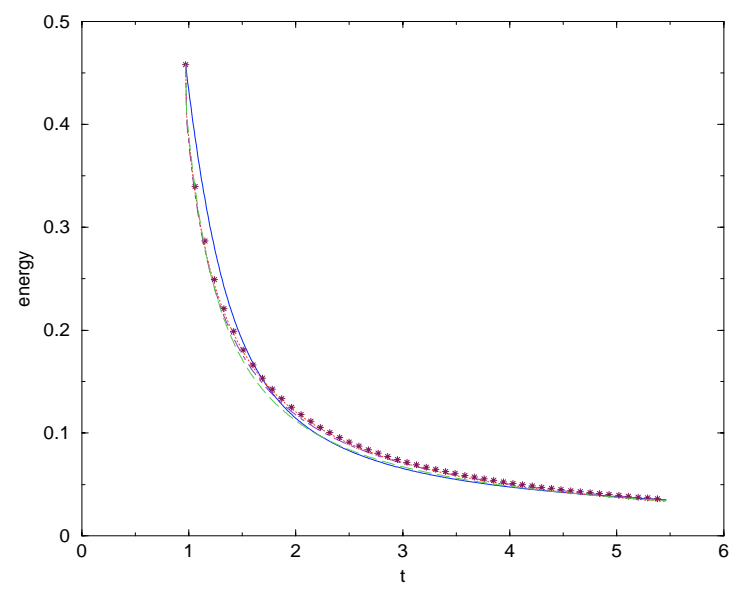

Figure 6. Energy decay: solid line: reference DNS filtered on $32^{3}$ modes; dashed line: Smagorinsky model with $C_{\mathrm{s}}=0.27$; dot-dashed line: selective Smagorinsky model, with $\beta_{0}=15^{0}$ and $C_{\mathrm{s}}=0.36$; stars: selective Smagorinsky model, with $\beta_{0}=5^{0}$ and $C_{\mathrm{s}}=0.29$; dotted line: selective anisotropic model with $C_{\mathrm{a}}=0.4$.

\section{Conclusion}

The problem of LES closure modeling was addressed in this paper from a mathematical and numerical standpoints. An analysis of the vorticity form of the Navier-Stokes equations and of the possible vorticity blow-up mechanisms provides some insight into the optimal form of subgrid-scale models seen as regularization terms for the Navier-Stokes equations.

This approach leads to two classes of models which have been proposed in the literature on physical or numerical grounds [14,18]: anisotropic models which distinguish between strain directions to dissipate energy and selective Smagorinsky models which dissipate only in flow regions where vorticity direction undergoes rapid variations. 


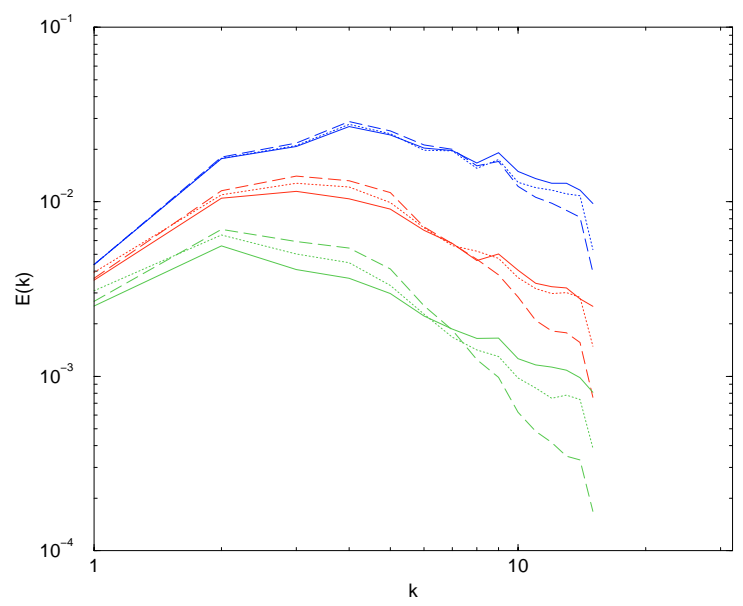

FiguRE 7. Energy spectrum at times $1.28,2.45$ and 4.49 (from top to bottom) for the reference DNS (solid line), LES with Smagorinsky (dashed line) and selective anisotropic (dotted line) models.

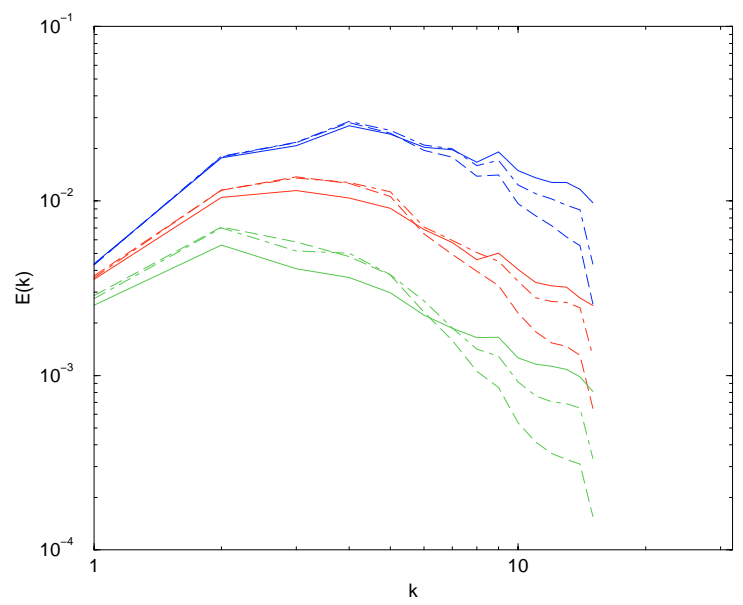

FiguRE 8. Energy spectrum at times $1.28,2.45$ and 5.46 for the reference DNS (solid) and LES with Smagorinsky (dashed) and selective Smagorinsky (dot-dashed) models.

Numerical tests on decaying turbulence reveal that both anisotropic and selective Smagorinsky model avoid to a large extent the excessive dissipation of the classical Smagorinsky model. The anisotropic model has already been proved to have also the correct asymptotic behavior near solid walls [14] and it would be interesting to extend the present comparison to channel flow configurations.

On the mathematical side, an interesting open question that remains to be addressed is the regularity of the Navier-Stokes equations complemented with the corresponding subgrid-scale terms.

Acknowledgements. The help of F. Bouchon in processing the turbulence data is gratefully acknowledged. The second author was partially supported by European Science Foundation (AMIF program). 


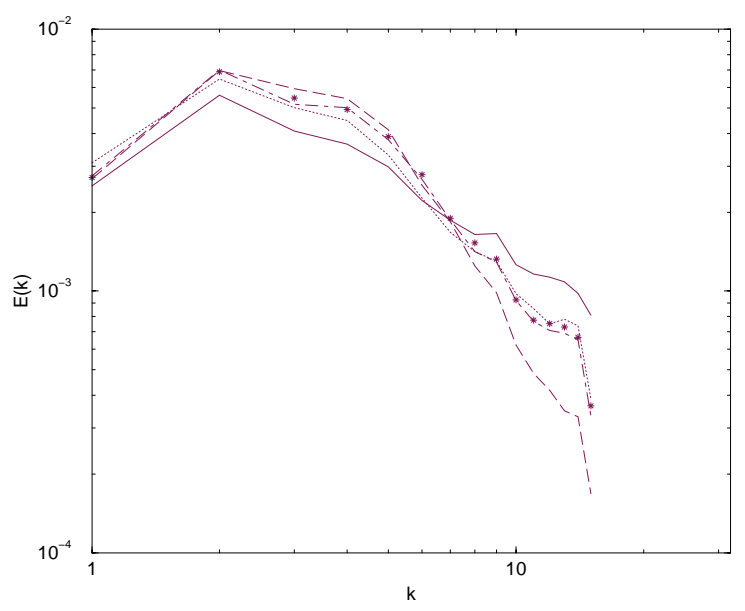

FIgURE 9. Spectra at $t=5.46$ for DNS and LES with classical and selective Smagorinsky models. Same captions as in Figure 6.

\section{REFERENCES}

[1] AGARD, A selection of test cases for the evaluation of large-eddy simulations of turbulent flows. Advisory report no. 345 (1998).

[2] J.T. Beale, T. Kato and A. Majda, Remarks on the Breakdown of Smooth Solutions for the 3D Euler Equations. SpringerVerlag, Comm. Math. Phys. 94 (1984).

[3] H. Beirão da Vega and L.C. Berselli, On the regularizing effect of the vorticity direction in incompressible viscous flows. Differential Integral Equations 15 (2002).

[4] V. Borue and S.A. Orszag, Local energy flux and subgrid-scale statistics in three dimensional turbulence. J. Fluid Mech. 366 (1998).

[5] C. Canuto, M.Y. Hussaini, A. Quarteroni and T.A. Zang, Spectral Methods in Fluid Dynamics. Springer-Verlag (1988).

[6] A. Chorin, Vorticity and Turbulence. Springer Verlag, Appl. Math. Sci. 103 (1994).

[7] R.A. Clark, J.H. Ferziger and W.C. Reynolds, Evaluation of subgrid scale models using an accurately simulated turbulent flow. J. Fluid Mech. 91 (1979).

[8] G. Comte-Bellot and S. Corrsin, Simple Eulerian time correlation of full and narrow-band velocity signals in grid-generated isotropic turbulence. J. Fluid Mech. 48 (1971).

[9] P. Constantin, Geometric Statistic in turbulence. SIAM Rev. 36 (1994).

[10] P. Constantin, Navier-Stokes Equations and Fluid Turbulence. Preprint arXiv:math.AP/0003235 (2000).

[11] P. Constantin and Ch. Fefferman, Direction of Vorticity and the Problem of Global Regularity for The Navier-Stokes Equations. Indiana Univ. Math. J. 42 (1993).

[12] P. Constantin, Ch. Fefferman and A. Majda, Geometric Constraints on Potentially Singular Solutions for the 3-D Euler Equations. Comm. Partial Differential Equations 21 (1996).

[13] P. Constantin and C. Foias, Navier-Stokes Equations. Univ. of Chicago Press, Chicago, Chicago Lectures in Math. (1989).

[14] G.-H. Cottet, Anisotropic Subgrid-Scale Numerical Schemes for Large Eddy Simulation of Turbulent Flows. Unpublished report (1997).

[15] G.-H. Cottet, D. Jiroveanu and B. Michaux, Simulation des grandes échelles : aspects mathématiques et numériques. ESAIM Proc. 11 (2002) 85-95.

[16] G.-H. Cottet and O.V. Vasilyev, Comparison of Dynamic Smagorinsky and Anisotropic Subgrid-Scale Models, in Proceedings of the Summer Program, Center for Turbulence Research (1998).

[17] G.-H. Cottet and A.A. Wray, Anisotropic grid-based formulas for subgrid-scale models, in Annual Research Brief, Center for Turbulence Research, Stanford University and NASA Ames Research Center (1997).

[18] E. David, Modélisation des écoulements compressibles et hypersoniques : une approche instationnaire. Ph.D. thesis, INPGLEGI Grenoble (1993).

[19] T. Dubois and F. Bouchon, Subgrid-scale models based on incremental unknowns for large eddy simulations, in Annual Research Brief, Center for Turbulence Research, Stanford University and NASA Ames Research Center (1998).

[20] F. Ducros, Simulation numérique directe et des grandes échelles de couches limites compressibles. Ph.D. thesis, INPG-LEGI Grenoble (1995). 
[21] C. Foias, D. Holm and E. Titi, The three dimensional viscous Camassa-holm equations, and their relation to the Navier-Stokes equations and turbulence theory. J. Dynam. Differential Equations 14 (2002).

[22] G.P. Galdi and W.J. Layton, Approximating the larger eddies in fluid motion II: a model for space filtered flow. Math. Models Methods Appl. Sci. 10 (2000).

[23] M. Germano, U. Piomelli, P. Moin and W.H. Cabot, A Dynamic Subgrid-Scale Eddy Viscosity Model. Phys. Fluids A 3 (1991).

[24] T. Iliescu and P. Fischer, Large Eddy Simulation of Turbulent Channel Flows by the Rational LES Model. Preprint ANL/MCSP932-0302 (2002).

[25] D. Jiroveanu, Analyse mathématique et numérique de certains modèles de viscosité turbulente. Ph.D. thesis, University Joseph Fourier Grenoble I (2002).

[26] A.N. Kolmogorov, The local structure of turbulence in incompressible viscous fluid for very large Reynolds numbers. Dokl. Akad. Nauk. SSSR 30 (1941).

[27] O.A. Ladyszenskaya, The Mathematical Theory of Viscous Incompressible Flow. Gordon and Breasch, New York (1969).

[28] O.A. Ladyszenskaya, On the Dynamical System Generated by the Navier-Stokes Equations. English translation in J. Sov. Math. 3 (1975).

[29] O.A. Ladyszenskaya, New equations for the description of the viscous incompressible fluids and solvability in the large of the boundary value problems for them, in Boundary Value Problems of Mathematical Physics V, Amer. Math. Soc., Providence, RI (1970).

[30] A. Leonard, Energy cascade in large-eddy simulations of turbulent flows. Adv. Geophysics 18 (1974).

[31] J. Leray, Sur le mouvement d'un liquide visqueux emplissant l'espace. Acta Math. 63 (1934).

[32] M. Lesieur, Turbulence in Fluids. Kluwer Academic Publishers, Dordrecht (1997).

[33] M. Lesieur and O. Metais, New trends in large eddy simulations of turbulence. Annu. Rev. Fluid Mech. 28 (1996) 45-82.

[34] J.L. Lions, Quelques méthodes de résolution des problèmes aux limites non-linéaires. Dunod, Paris (1969).

[35] S. Liu, C. Meneveau and J. Katz, On the properties of similarity subgrid-scales models as deduced from measurements in a turbulent jet. J. Fluids Mech. 275 (1994) 83-119.

[36] J. Malek and J. Nečas, A Finite-Dimensional Attractor for Three-Dimensional Flow of Incompressible Fluids. J. Differential Equations 127 (1996).

[37] J. Marsden and S. Skholler, Global Well-posedness for the Lagrangian Averaged Navier-Stokes (LANS) Equations on Bounded Domains. Meeting of Royal Society London (2000).

[38] J. Marsden, T. Ratiu and S. Skholler, The Geometry and Analysis of the Averaged Euler Equations and a New Diffeomorphism Group. Geom. Funct. Anal. 10 (2000).

[39] O. Metais and M. Lesieur, Spectral large-eddy simulation of isotropic and stably stratified turbulence. J. Fluid Mech. 239 (1992) 157-194.

[40] K. Mosheini, S. Skholler, B. Kosovič, J. Marsden, D. Caratti, A. Wray and R. Rogallo, Numerical Simulations of Homogeneous Turbulence Using the Lagrangian Averaged Navier-Stokes Equations in Proc. CTR summer school (2000).

[41] C. Parès, Uniqueness and regularity of solution of the equations of a turbulence model for incompressible fluids. Appl. Anal. 43 (1992).

[42] U. Piomelli, Y. Yu and R.J. Adrian, Subgrid scale energy transfer and near-wall turbulence structure. Phys. Fluids 8 (1996) $215-224$.

[43] R.S. Rogallo and P. Moin, Numerical Simulation of Turbulent Flows. Annu. Rev. Fluid Mech. 16 (1984) 99-137.

[44] J. Smagorinsky, General circulation experiments with the primitive equations. I. The basic experiment. Monthly Weather Review 91 (1963).

[45] C.G. Speziale, Turbulence modeling for time-dependent RANS and VLES: A review. AIAA Journal 36 (1998).

[46] S. Stoltz and N.A. Adams, An Approximative Deconvolution Procedure for Large-Eddy Simulation. Phys. Fluids 11 (1999).

[47] R. Temam, Infinite Dimensional Dynamical Systems in Mechanics and Physics. Springer-Verlag, Appl. Math. Sci. 68 (1988).

[48] B. Vreman, Direct and large-eddy simulation of the compressible turbulent mixing layer. Ph.D. thesis, University of Twente (1995).

[49] D.C. Wilcox, Turbulence Modeling CFD. DCW Industries Inc. (1993).

[50] E. Zeidler, Nonlinear Functional Analysis and its Applications. Springer-Verlag (1990).

To access this journal online:

www.edpsciences.org 